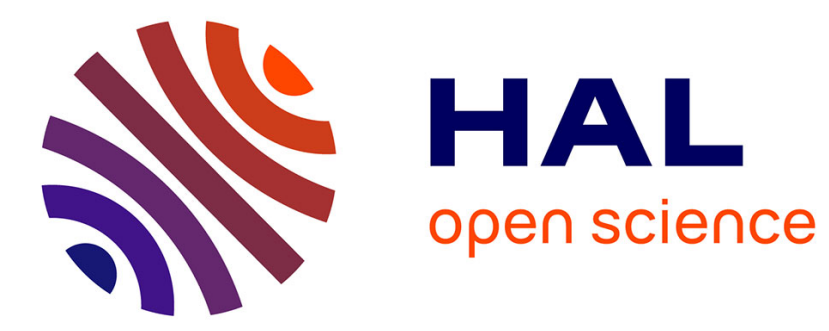

\title{
A model based 2-DOF fault tolerant control strategy
}

\author{
Tushar Jain, Joseph Julien Yamé, Dominique Sauter
}

\section{To cite this version:}

Tushar Jain, Joseph Julien Yamé, Dominique Sauter. A model based 2-DOF fault tolerant control strategy. 18th Mediterranean Conference on Control and Automation, MED'10, Jun 2010, Marrakech, Morocco. pp.1073-1078. hal-00547952

\section{HAL Id: hal-00547952 \\ https://hal.science/hal-00547952}

Submitted on 17 Dec 2010

HAL is a multi-disciplinary open access archive for the deposit and dissemination of scientific research documents, whether they are published or not. The documents may come from teaching and research institutions in France or abroad, or from public or private research centers.
L'archive ouverte pluridisciplinaire HAL, est destinée au dépôt et à la diffusion de documents scientifiques de niveau recherche, publiés ou non, émanant des établissements d'enseignement et de recherche français ou étrangers, des laboratoires publics ou privés. 


\title{
A model based 2-DOF fault tolerant control strategy
}

\author{
Tushar Jain, Joseph J. Yamé, Dominique Sauter
}

\begin{abstract}
In this paper, a novel concept of model based fault tolerance control (FTC) is presented. The FTC is achieved by 2-DOF control strategy: feedback control and feed forward control. Robustness issues are handled by the optimal feedback control and the time varying fault behavior by feed forward path. Firstly, a fault diagnosis scheme is presented for detecting and estimating the fault behavior from the observer based residual generator. The estimated behavior of the fault is used by the feed-forward control algorithm to make appropriate changes in the manipulated variable which keeps the controlled variable near to its set value. The effectiveness of the proposed scheme is analyzed using behavioral theoretic approach.
\end{abstract}

\section{INTRODUCTION}

FAULT is an unknown dynamical behavior that changes the behavior of systems in such a way it no longer satisfies its purpose. In order to avoid production deteriorations or damage to machines and humans, fault has to be found as quickly as possible and decisions that stop the propagation of their effects have to be made. The aim is to make the system fault tolerant. If they are successful, the system function is satisfied also after the appearance of a fault, possibly after a short time of degraded performance. The control algorithm adapts to the faulty plant and the overall system satisfies its function again. A generic structure of FTC systems is that which incorporates the supervisory level with the usual feedback loop [1]. The supervisory level constitutes the fault detection and estimation block that helps to determine the new control law to provide the stability. It can be achieved by various control re-design techniques: fault accommodation [2] and controller re-configuration [3]. The structure of controller is fixed in the former approach while it is not fixed in the later. In the aforesaid approaches, work is mainly concentrated on the model-free FTC and no fault detection analysis is carried out explicitly. On the other side with model based approaches, (Fault Diagnosis) FD provides the information of the fault and the supervisory level takes care of its behavior affecting the system. The basic idea of model-based FD is to generate analytical redundancy with the help of mathematical model of supervised systems. Observer based FD is one of the most important kinds of model-based FD approaches [1, 4]. Various observer based schemes are discussed in [4]: Luenberger observer, Unknown input observer (UIO), Eigen structure assignment. The residual generated by the observer

Manuscript received February 4, 2010

Tushar Jain, Dominique Sauter and Joseph J. Yamé are with Centre de Recherche en Automatique de Nancy - CRAN-UMR 7039, Nancy Université, CNRS-INPL-UHP, France (e-mail: tushar.jain@cran.uhpnancy.fr). based FD, then feeds to the fault estimation block to determine the fault occurrence time and its magnitude.

On the other hand, feed-forward control is best deployed in control systems design applications where the process or controlled variable behavior is well understood. It is also very useful in designs where the process is not understood at all, but the behavior of the process can be measured and experience has shown that it is replicable under known operating points. In a feed-forward control there is a coupling from the set point and/or from the unknown signal affecting the system directly to the control variable [5]. Here unknown signals focus the faults acting on the system. A feed-forward path generally employed to cancel out the effect of known disturbances. But here the novelty of the approach lies in the use for unknown faults. The fault information generated by fault detection estimation procedure is very useful to FTC. However, links between fault diagnosis and FTC techniques are still lacking [6]. Some results on the integration of FDI with FTC can be found in $[7,8]$.

In this paper, we present a 2-DOF fault tolerant control strategy. The optimal feedback controller takes care of the stability issues for an unstable system while the feedforward controller handles the issue of settling the effect of fault on the behavior of system. From the basic control theory [9], the feed-forward path does not affect the closed loop system stability. This is usually referred to as the second degree of freedom in control loops. More industrial applications of feed-forward controller can be studied from [10]. The proposed approach is explained by the mathematical framework of behavioral systems [11, 12] and the FD technique [13] depicting the correlation between the parity based analytical redundancy relation (ARR) and the observer based approaches. The scheme is illustrated with a multi input-multi output system.

\section{MATHEMATICAL FRAMEWORK}

In this section, the theory behind the novel scheme is explained with the help of behavioral system approach [11]. From the behavioral perspective, a dynamical system can be considered as a collection of time trajectories which maps input signals to output signals.

Definition 1: A dynamical system $\Sigma$ is represented by a triple $\Sigma=(\mathbb{T}, \mathbb{S}, \mathcal{B})$ where $\mathbb{T} \subseteq \mathbb{R}$, called the time axis, $\mathbb{S}$ a set called the signal space and $\mathcal{B} \subseteq \mathbb{S}^{\mathbb{T}}$ called the behavior. $\left(\mathbb{S}^{\mathbb{T}}\right.$ is the set of all $\mathbb{S}$-valued time trajectories). Reflecting this broad framework, a dynamical system includes three ingredients: first, a set $\mathbb{T}$ that is interpreted as a mathematical 
model of time. Second, a set $\mathbb{S}$ in which the signals take their values. Thus a trajectory is a function

$$
s: \mathbb{T} \rightarrow \mathbb{S}, \quad t \mapsto s(t)
$$

By $\mathbb{S}^{\mathbb{T}}$, we denote the set of all functions that are defined on $\mathbb{T}$ and take their values in $\mathbb{S}$. The third and the most important part of the definition is the set $\mathcal{B} \subseteq \mathbb{S}^{\mathbb{T}}$ formalizing the set of signals that can occur in the system i.e. which obey the laws that governs the system.

Consider Fig.1, the behavior $\mathcal{B}_{\text {spec }}$ is a subset of the space $\mathbb{T} \times \mathbb{S}$. For a plant $\mathcal{P}$, the system dynamics, according to Def. 1 is represented by $\Sigma_{\mathcal{P}}=\left(\mathbb{T}, \mathbb{S}, \mathcal{B}_{\mathcal{P}}\right) . \mathcal{B}_{\text {spec }}$ defines that the system should follow these trajectories for optimal tracking. Now from Fig.1 (a), it is clear that the plant dynamics is a partial subset of $\mathcal{B}_{\text {spec }}$. To make it working in the stable mode or to follow a desired behavior, a controller $\mathcal{C}$ for the plant $\mathcal{P}$ with dynamical system $\Sigma_{\mathcal{C}}=\left(\mathbb{T}, \mathbb{S}, \mathcal{B}_{\mathcal{C}}\right)$ is defined. When the plant and the controller are connected, the interconnected system is denoted by $\Sigma_{\mathcal{P}} \cap \Sigma_{\mathcal{C}}$. Thus the plant signals are forced to obey the laws of both plant and the controller simultaneously. The combined behavior comprising the set of trajectories $y: \mathbb{T} \rightarrow \mathbb{S}$ that are compatible with the laws of $\Sigma \mathcal{P}$ and $\Sigma \mathcal{C}$ is given by

$$
\Sigma_{\mathcal{P}} \cap \Sigma_{\mathcal{C}}=\left(\mathbb{T}, \mathbb{S}, \mathcal{B}_{\mathcal{P}} \cap \mathcal{B}_{\mathcal{C}}\right) \subseteq \Sigma_{\mathrm{spec}}
$$

where $\Sigma_{\text {spec }}$ is the desired dynamical system

For a fault $f$ acting on the plant, Fig.1 (b) gives the plant behavior and it changes to $\mathcal{B}_{f}$. The control objective requirement (2) now may no longer be satisfied by the current controller because of the interconnection is not a subset of $\mathcal{B}_{\text {spec }}$ anymore. This problem is handled in [3] i.e.,

$$
\mathcal{B}_{\text {spec }}=\mathcal{B}_{\mathcal{P}} \cap \mathcal{B}_{\mathcal{C}}{ }^{\text {new }} \neq \emptyset
$$

by real-time model free reconfiguration mechanism following the unfalsified control concept [14]. Fig.1 (c) shows that approach. In our current strategy, instead of reconfiguring the control structure, emphasis is given on retaining the behavior of the system with an additional feedforward control. This takes care of the overall system to obey the desired behavior in the interconnected form.

Proposition 2: A necessary and sufficient condition for applying 2-DOF control is that the plant affected by fault should not become inconsistent with the specified behavior.

Fig.1 (d) gives the combined system behavior following the current approach. A feed-forward controller $\mathcal{C}^{\text {ff }}$ with dynamical representation $\Sigma_{C} f f=\left(\mathbb{T}, \mathbb{S}, \mathcal{B}_{C}{ }^{f f}\right)$ for the combined system given by $\Sigma_{f} \cap \Sigma_{\mathcal{C}}$ is designed to follow the desired trajectories i.e.

$$
\Sigma_{f} \cap \Sigma_{\mathcal{C}} \cap \Sigma_{\mathcal{C}}^{f f}=\left(\mathbb{T}, \mathbb{S}, \mathcal{B}_{f} \cap \mathcal{B}_{\mathcal{C}} \cap \mathcal{B}_{\mathcal{C}}^{f f}\right) \subseteq \Sigma_{\text {spec }}
$$

Now considering the fault tolerant control problem for the plant subjected to faults. The effect of fault occurring on the system changes its behavior and following the above proposition the desired specification is regained using the 2DOF strategy.

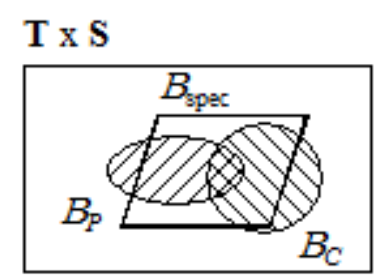

(a)

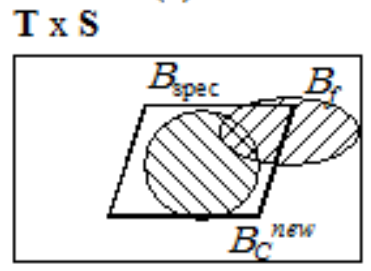

(c)

\section{$\mathbf{T} \times \mathbf{S}$}

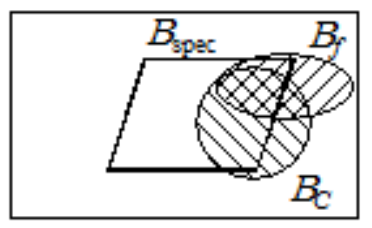

(b)

$\mathbf{T} \times \mathbf{S}$

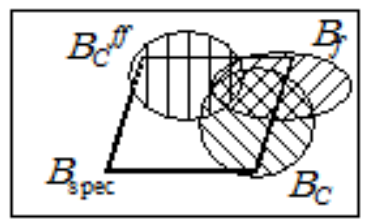

(d)
Fig.1: (a) Control of faultless system; (b) Effect of fault on system; (c) Control reconfiguration [3]; (d) Feed-forward control approach

\section{2-DOF CONTROL CONFIGURATION}

The implementation procedure of the proposed approach is shown in Fig.2. From the control theoretic point of view, FTC is an interaction between the system and the controller. The plant is subjected to faults and disturbances. There are number of ways for designing the observer-based residual generator mentioned in literature [4]. In this configuration, the observer based residual generator is inspired from the approach followed in [13]. The generated residual and the state error feed to the fault estimation block to determine the magnitude and the fault occurrence time. Now based upon the information available of the type of fault, feed-forward controller compensates its effect. This monitors the system to regain its desired behavior subjecting to fault.

Consider a continuous linear time-invariant (LTI) system describe by

$$
\begin{aligned}
& \dot{x}(t)=A x(t)+B u(t)+E_{x} d(t)+F_{x} f(t) \\
& y(t)=C x(t)+D u(t)+E_{y} d(t)+F_{y} f(t)
\end{aligned}
$$

where $x \in R^{n}$ denotes the state vector, $u \in R^{k_{u}}$ the control input vector, $y \in R^{m}$ the measured output vector, $d \in R^{k_{d}}$ the disturbance vector, $f \in R^{k_{f}}$ the fault vector, $A \in R^{n \times n}, B \in R^{n \times k_{u}}, C \in R^{m \times n}, D \in R^{m \times k_{u}}$, $E_{x} \in R^{n \times k_{d}}, E_{y} \in R^{n \times k_{d}}, F_{x} \in R^{n \times k_{f}}, F_{y} \in R^{m \times k_{f}}$ are constant matrices of compatible dimensions. For residual generator, the observer [15] can be designed as

$$
\begin{aligned}
& \dot{z}(t)=G z(t)+J u(t)+L y(t) \\
& r(t)=w z(t)+p u(t)+v y(t)
\end{aligned}
$$

The conditions to be satisfied by observer matrices are: 


$$
\begin{aligned}
& T A-G T=L C, \quad v C+w T=0 \\
& T B-L D=J, \quad p+v D=0
\end{aligned}
$$

where $G$ is a stable matrix. The error between the original states and the estimated states is given by

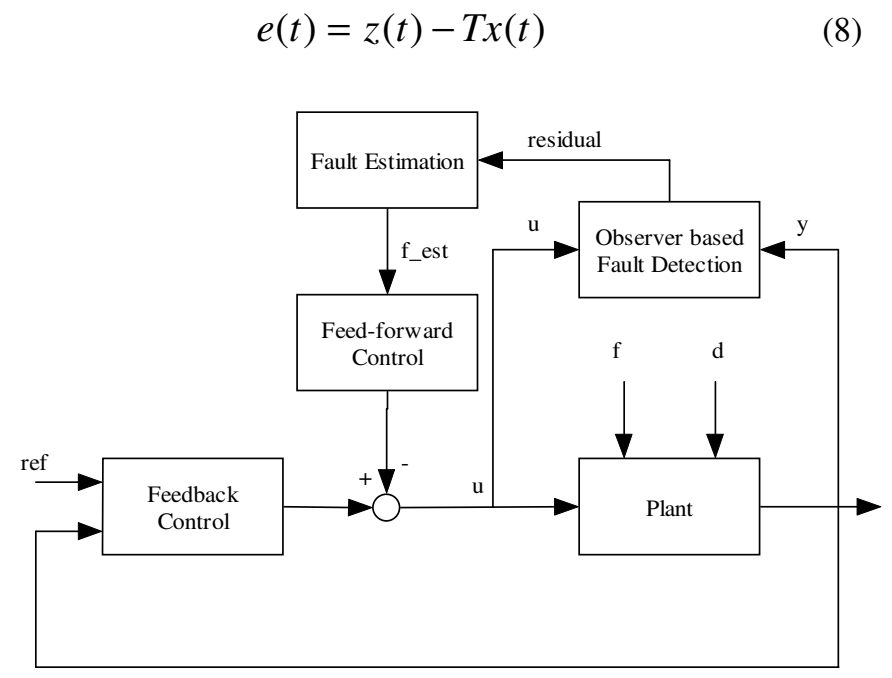

Fig.2 2-DOF FTC implementation

Following the approach described in $[13,16]$ for computing (7) by parity space approach, disturbance is decoupled that makes residual insensitive to it and react only to faults. Firstly, three matrices $H_{0}$ (depending on $A, C$ ), $H_{u}$ (depending on $A, B, C, D$ ), and $H_{d}$ (depending on $A, E_{x}, C$, $E_{y}$ ) are defined as

$$
\begin{gathered}
H_{o}=\left[\begin{array}{c}
C \\
C A \\
\vdots \\
C A^{q}
\end{array}\right], \quad H_{u}=\left[\begin{array}{cccc}
D & 0 & 0 & 0 \\
C B & D & \ddots & 0 \\
\vdots & \ddots & \ddots & 0 \\
C A^{q-1} B & \cdots & C B & D
\end{array}\right] \\
H_{d}=\left[\begin{array}{cccc}
E_{y} & 0 & 0 & 0 \\
C E_{x} & E_{y} & \ddots & 0 \\
\vdots & \ddots & \ddots & 0 \\
C A^{q-1} E_{x} & \cdots & C E_{x} & E_{y}
\end{array}\right]
\end{gathered}
$$

where $q$ is an integer, $H_{0} \in R^{m(q+1) \times n}, H_{u} \in R^{m(q+1) \times k_{u}(q+1)}, H_{d} \in R^{m(q+1) \times k_{d}(q+1)}$

A vector $v_{q}$ is given such that it satisfies

$$
\begin{aligned}
& v_{q}\left(\left[H_{o} H_{d}\right]\right)=0 \\
& \rho_{q}=v_{q} H_{u}
\end{aligned}
$$

Portioning the two newly introduced vectors gives:

$$
\begin{aligned}
& v_{q}=\left[\begin{array}{llll}
v_{q, 0} & v_{q, 1} & \cdots & v_{q, q}
\end{array}\right], \quad v_{q, i} \in R^{1 \times m} \\
& \rho_{q}=\left[\begin{array}{llll}
\rho_{q, 0} & \rho_{q, 1} & \cdots & \rho_{q, q}
\end{array}\right], \quad \rho_{q, i} \in R^{1 \times k_{u}}
\end{aligned}
$$

Further the other matrices in (7) are given on the basis of (9) and (11) as:

$$
\begin{aligned}
& T=\left[\begin{array}{cccc}
v_{q, 1} & \cdots & v_{q, q-1} & v_{q, q} \\
v_{q, 2} & \cdots & v_{q, q} & 0 \\
\vdots & \ddots & \ddots & \vdots \\
v_{q, q} & 0 & \cdots & 0
\end{array}\right]\left[\begin{array}{c}
C \\
C A \\
\vdots \\
C A^{q-1}
\end{array}\right] \\
& G=\left[\begin{array}{cccc}
0 & \cdots & 0 & g_{1} \\
1 & \cdots & 0 & g_{2} \\
\vdots & \ddots & \ddots & \vdots \\
0 & \cdots & 1 & g_{q}
\end{array}\right], \quad J=\left[\begin{array}{c}
\rho_{q, 0} \\
\rho_{q, 1} \\
\vdots \\
\rho_{q, q-1}
\end{array}\right]+\left[\begin{array}{c}
g_{1} \\
g_{2} \\
\vdots \\
g_{q}
\end{array}\right] \rho_{q, q} \\
& L=-\left[\begin{array}{c}
v_{q, 0} \\
v_{q, 1} \\
\vdots \\
v_{q, q}
\end{array}\right]-\left[\begin{array}{c}
g_{1} \\
g_{2} \\
\vdots \\
g_{q}
\end{array}\right] v_{q, q}, \quad w=-1 * \operatorname{eyc}(q) \\
& p=\left[\begin{array}{llll}
-\rho_{q, q} & -\rho_{q, q} & \cdots & -\rho_{q, q}
\end{array}\right]^{T}, \quad v=\left[\begin{array}{llll}
v_{q, q} & v_{q, q} & \cdots & v_{q, q}
\end{array}\right]^{T}
\end{aligned}
$$

where $g_{1}, g_{2}, \cdots, g_{q}$ are free-selectable constants which stabilize the matrix $G$. For the selection of $q$, [1] defines the condition such that

$$
\operatorname{rank}\left(H_{x} \quad H_{d}\right)<(q+1) m
$$

In this case, the residual dynamics and error is governed by

$$
\begin{aligned}
& \dot{e}(t)=G e(t)+\left(L F_{y}-T F_{x}\right) f(t) \\
& r(t)=w e(t)+v F_{y} f(t)
\end{aligned}
$$

When the faults are presents, one gets eq. 13 with disturbance effects decoupled from the residual. In order for the fault to be detected, the matrix associated to it should be non-zero. This approach is very different from unknown input observer (UIO) because in UIO, the design of observer depends on the knowledge of system matrices in state space while here it merely depends on $H_{0}, H_{u}$. So from this perspective, utilizing the approach in [16], these matrices can be easily computed from the filtered data. In that case, the overall system is given by the new augmented vector [e(t) $r(t)$ ]', where $e(t)$ is the states, $r(t)$ is the output, and $f(t)$ is an unknown input to the system. The states for this new system are evaluated by the method described in [18]. Considering only the actuator faults at this stage, eliminates the need of fault isolation step. In that case, $F_{y}$ becomes zero 
and (13) can be written as

$$
\begin{aligned}
\dot{e}(t) & =G e(t)-T F_{x} f(t) \\
r(t) & =w e(t)
\end{aligned}
$$

So from (14) $T F_{x} \neq 0$. In particular, the residual $r(t)$ will be sensitive to the fault in the $i$ th actuator iff $T b_{i} \neq 0$, where $b_{i}$ is the $i$ th column of the input matrix $B$. Similarly, the residual can be made sensitive to sensor fault. Since the sensor fault vector has the direct impact on the residual output, and then sensor faults can also be detected. Differentiate (14.b) and using (14.a), results in

$$
\dot{r}(t)=w\left(G e(t)-T F_{x} f(t)\right)
$$

Corollary 1: Let $A \in \mathbb{F}^{n x m}$. Then, $A$ is left invertible if there exists a matrix $A^{\mathrm{L}} \in \mathbb{F}^{n x m}$ such that $A^{\mathrm{L}} A=I_{m}$

Using (15)-(14.b) and satisfying cor.1, we get

$$
\hat{f}(t)=\left(T F_{x}\right)^{-1}\left[G w^{-1} r(t)-w^{-1} \dot{r}(t)\right]
$$

If we consider about the actuator fault, then $F_{x}$ is given by $B$, and it should have full column rank. To achieve this goal, assumption is given by

Assumption 1: $\operatorname{rank}\left(w T F_{x}\right)=\operatorname{rank}\left(T F_{x}\right)$

Under full state measurements $(w=I)$ the above assumption is clearly satisfied.

The derivative of residual does not affect in the estimation of fault if we consider about the amplification of noise due to differentiation. The residual is insensitive to disturbances because of the decoupling used in (10). This can be an advantage compared to estimation of fault by using the derivative of output [17] as the output may be affected by high frequency component. Now using (16), the actuator fault can be estimated. The estimated knowledge of the fault is given to the feed-forward path, which makes appropriate changes in the manipulated variable to keep the controlled variable near its set point. The feed-forward is used along with feedback controller to control the multi-variable system.

In the further section, the effectiveness of using behavioral theory in achieving the desired behavior is depicted by an example. It is shown that the behavior of system changes upon acting the fault and the forward path controller does not provide correction instantaneously though it achieved at $t \rightarrow \infty$. The system is still affected by the fault and no measures are taken to lower its effect. This scenario is shown in Fig.1 (b). The objective is to lower down the effects of fault in the output. Using the notion of behavior introduced in previous section, we show how such inference can be made. At this stage, it is worth noting that the set $\mathcal{B}_{\mathcal{P}}$ considers all signals which can occur as the outcome of plant, $\mathcal{P}$. With this known set, it is possible to verify if a potential feedback controller $\mathcal{C}$ would have implemented a closed loop system $\Sigma \mathcal{P} \cap \Sigma \mathcal{C}$ satisfying the performance goal through the test

$$
\mathcal{B}_{\text {spec }} \subseteq \mathcal{B}_{\mathcal{P}} \cap \mathcal{B}_{\mathcal{C}} \neq \varnothing
$$

This means that controller $\mathcal{C}$ satisfy the performance objective. Now consider a fault occur on the system and the behavior of the plant changes to $\mathcal{B}_{f}$. Then the behavior defined by (17) no more is valid. To regain the desired behavior of the system, a feed-forward control is applied for compensating the effect of fault. The full behavior is governed by

$$
\mathcal{B}=\left\{s=(e, u, \widehat{f}) \in \mathbb{S}^{\mathbb{T}}: u=C^{f p} e-C^{f f} \widehat{f}\right\}
$$

where $e$ is the error given by the difference of output and reference, $C^{f p}$ and $C^{f f}$ is the forward path and feed-forward controller respectively. Graphically, it is shown in Fig.1 (d). Here by feedback controller and forward path controller, we mean the same as it only results in closed loop control providing stability to the faultless system. The forward path controller is an optimal controller designed using $L Q R$ method explained in next section. This makes the system stable prior to action of faults. As the fault occurs on the system, it no longer satisfies the goal. The fault is detected and the knowledge of estimated fault modifies the control input resulting in follow the desired behavior. The control law is changed through the feed-forward path achieving fault accommodation. It is given by

$$
u(t)=u_{0}(t)-M \hat{f}(t)
$$

where $M=B^{-1} F_{x}$ and $u_{0}(t)$ denotes the robust control policy.

\section{EXAMPLE}

To illustrate the algorithm, consider an example of multi input-multi output system. System is subjected to single actuator fault and disturbances. The model is represented as

$$
\begin{aligned}
& \dot{x}(t)=\left[\begin{array}{ccc}
0 & 0.5 & 1 \\
-1 & -1 & 0.25 \\
1 & 0.25 & 2
\end{array}\right] x(t)+\left[\begin{array}{ll}
1 & 1 \\
1 & 0 \\
0 & 1
\end{array}\right] u(t)+\left[\begin{array}{l}
1 \\
1 \\
1
\end{array}\right] d(t) \\
& y(t)=\left[\begin{array}{lll}
1 & 0 & 0 \\
0 & 0 & 1
\end{array}\right] x(t)+\left[\begin{array}{l}
1 \\
1
\end{array}\right] d(t)
\end{aligned}
$$

The system in its current form is unstable. The feedback controller is designed using linear quadratic regulator (LQR) synthesis method. Note that since the LQR method results in pure state-feedback, integral action is added to the controller's structure in order to force the steady state error to zero. The design parameters are the weighting matrices $Q$ and $R$ of the performance index $\mathcal{J}=\underline{\int}\left(z^{T} Q z+u^{T} R u\right)$. These 
weighting matrices are obtained after subsequent iterations to achieve an acceptable tradeoff between performance and control effort. $Q$ and $R$ taken as diag[0.5 00.5$]$ and diag[1 1 [ respectively. The characteristic defining the system are: reference for output-1 is 1 unit whiles its 2 units for output2 ; sinusoidal disturbance is applied with amplitude 0.1 and frequency $30 \mathrm{rad} / \mathrm{sec}$..

It is seen that $q=3$ using (12.b). By choosing $g_{1}=-6, g_{2}$ $=-11, g_{3}=-6$, so that eigen values lies at $-1,-2,-3$, an observer based FD system is obtained as

$$
\begin{array}{r}
\dot{z}(t)=\left[\begin{array}{ccc}
0 & 0 & -6 \\
1 & 0 & -11 \\
0 & 1 & -6
\end{array}\right] z(t)+\left[\begin{array}{cc}
0.5870 & 0.5583 \\
0.1718 & 0.4581 \\
-0.2291 & 5.57 e-17
\end{array}\right] u(t) \\
+\left[\begin{array}{ll}
-1.4030 & 2.1904 \\
-2.5913 & 2.9921 \\
-1.3744 & 1.3744
\end{array}\right] y(t) \\
r(t)=\left[\begin{array}{ccc}
-1 & 0 & 0 \\
0 & -1 & 0 \\
0 & 0 & -1
\end{array}\right] z(t)+\left[\begin{array}{ll}
-0.2291 & -0.2291 \\
-0.2291 & -0.2291 \\
-0.2291 & -0.2291
\end{array}\right] y(t) \quad(21)
\end{array}
$$

The error between the estimated states and the original states is given by

$$
e(t)=z(t)-\left[\begin{array}{ccc}
0.3579 & 0.2291 & 0.2004 \\
0.2291 & -0.0573 & 0.2291 \\
-0.2291 & 0 & 0.2291
\end{array}\right] x(t)
$$
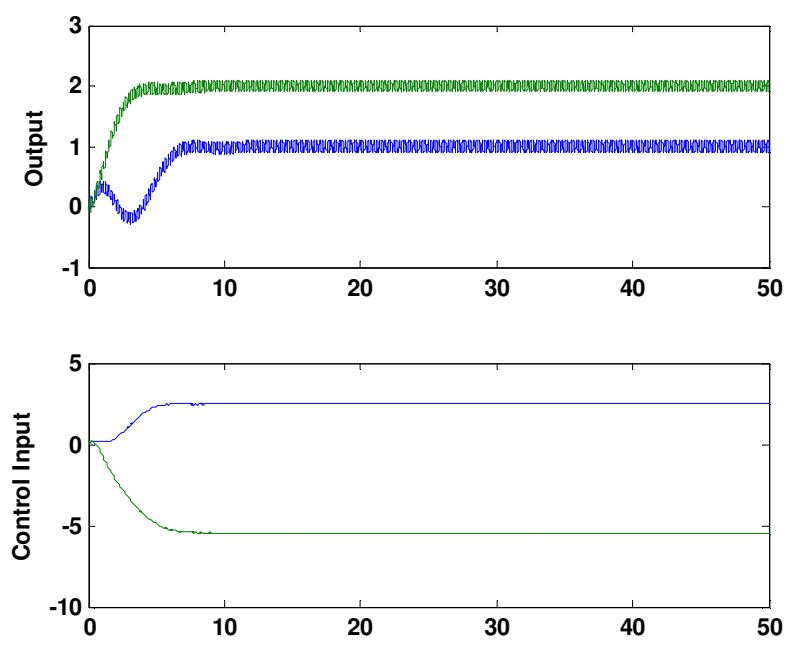

Fig. 3 System subjected to disturbances.

It can be easily verified from Fig. 3 that the forward path controller satisfies the desired behavior in faultless case. Now a constant fault in actuator- 1 is applied with step input at $t=10 \mathrm{sec}$ and magnitude 15 units. The system behavior subjected to fault and disturbance is shown in Fig.4 without feed-forward FTC.
Fig.4 (c) shows the non-zero residual after the fault acts on the system and change in optimal behavior of the system is shown in Fig.4 (a).
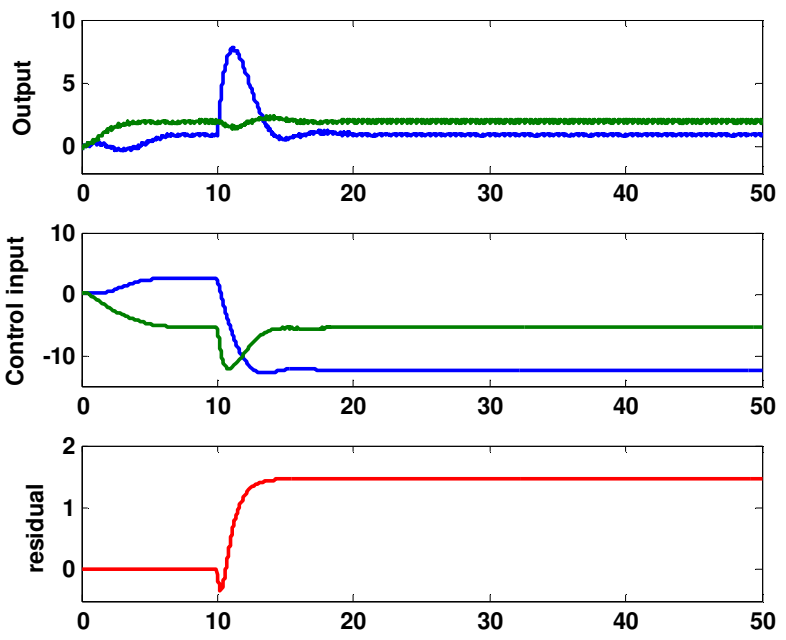

Fig.4 (a) Outputs; (b) control input; (c) residual

Referring to section III for estimation of fault and feedforward control and satisfy cor.1 and assumption gives Fig.5
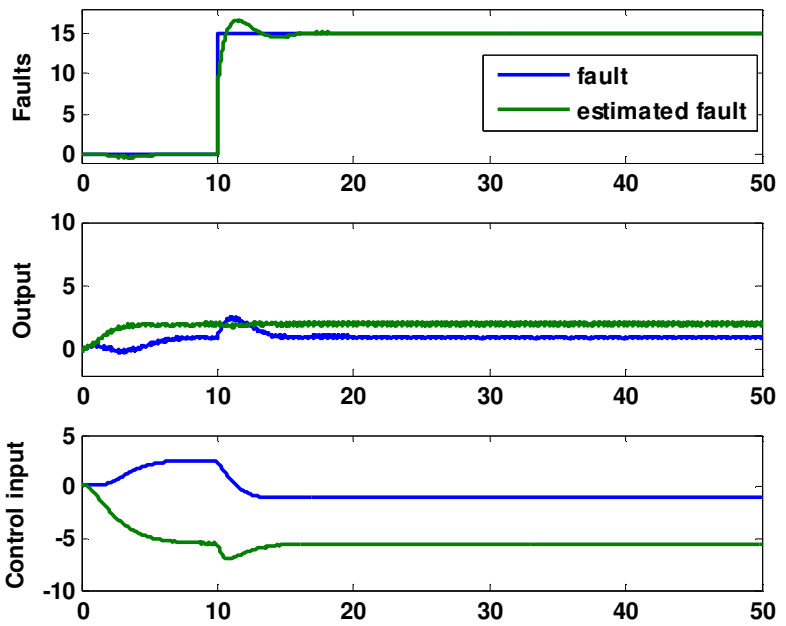

Fig.5 (a) Estimated Fault; (b) outputs; (c) control input

The effect of fault occurring on the output is lowered down by the feed-forward path as shown in Fig.5 (b). The timing issues regarding the detection delay and the fault estimation are not a point of limitation as the feed-forward path is always active in the loop.

\section{CONCLUSION}

In this paper, a 2-DOF approach to fault tolerant control is presented. A unifying mathematical framework is presented using behavioral theoretic approach. The work is in its initial stage and further research work includes two aspects. The first one is to analyze the different behavior of fault and more precise estimation of fault which can guarantee the 
faster recovery. Though the model-based approach is carried out here, the work is going in the direction of using model free concept. The observer design mainly depends on (11); if the vectors used in (11) can be computed from the inputoutput data then the knowledge about model parameters will not be required. Also, the fault isolation stage is still to be implemented as only the actuators faults are considered in the current work.

\section{REFERENCES}

[1] Blanke M., Kinnart M., Lunze J., Staroswiecki, "Diagnosis and FaultTolerant Control”, Springer-Verlag ,Berlin, 2003

[2] Yamé J.J., Kinnaert M., "A Fault Accommodation Strategy based on Closed-Loop Performance Monitoring", Proc. of IEEE Conf. on Decision and Control, Bahamas, 2004, pp. 5242-5247.

[3] Yamé J.J., Sauter D., "A Real-Time Model-Free Reconfiguration Mechanism for Fault-Tolerance: Application to a Hydraulic Process", Int. Conf. on Control, Automation, Robotics and Vision, Vietnam, 2008, pp. 91-96.

[4] Chen J., Patton R.J., "Robust Model-based Fault Diagnosis for Dynamic Systems", Kluwer Academic Publishers, Boston, 1999.

[5] Love J., "Process Automation Handbook: A guide to theory and practice", Springer-Verlag, London, 2007.

[6] Jiang J., Zhao Q., "Fault tolerant control system synthesis using imprecise fault identification and reconfiguration control", Proc. of IEEE Intl. Sym. on Intelligent Control, Gaithersburg, 1998, pp.169174

[7] Chen J., Patton R.J., Chen Z., "An LMI approach to fault-tolerant control of uncertain systems", Proc. of IEEE Intl. Sym. on Intelligent Control, Gaithersburg, 1998, pp.175-180.

[8] Mhaskar P., Gani A., El-Farra N.H., McFall C., Christodes P.D., Davis J.F., "Integrated fault-detection and fault-tolerant control for process systems", AIChE Journal, Vol.52,No.6, pp. 2129-2148, 2006.

[9] Levine W.S., "The Control Handbook", IEEE/CRC Press, 1996.

[10] Goodwin G.C., Graebe S.F., Salgado M.E., "Control System Design", Prentice Hall, 2000.

[11] Polderman J.W., Willems J.C., "Introduction to Mathematical Systems Theory': A behavioral approach", Springer-Verlag, NewYork, 1998.

[12] Willems J.C., "The Behavioral Approach to Open and Interconnected Systems: Modeling by Tearing, Zooming and Linking", IEEE Control Systems Magazine, December 2007, pp.46-99.

[13] Ding S.X., Ding E.L., Jeinsch T., "A numerical approach to optimization of FDI systems", Proc. of $37^{\text {th }}$ IEEE CDC, USA, pp. 1137-1142, 1998.

[14] Safonov M.G., Tsao T-C., "The Unfalsified Control Concept and Learning”, IEEE Trans. on Automatic Control, Vol.42, No.6, 1997, pp. 843-847.

[15] Frank P.M., Ding S.X., Marcu T., "Model-based Fault Diagnosis in Technical Process", Transactions of the Institute of Measurement and Control, Vol.22, No.1, 2000, pp. 57-101.

[16] Garnier H., Mensler M., Richard A., "Continuous-time Model Identification from Sampled-data: Implementation issues and Performance Evaluation", International Journal of Control, 76(13), pp. 1337-1357, 2003

[17] Park Y., Stein J.L., "Closed loop state and input observer for systems with unknown inputs", International Journal of Control, Vol.48, 1988, pp.1121-1136.

[18] Corless M.J., Tun, "State and Input estimation for a class of Uncertain Systems", Automatica, Vol.34, pp. 757-764, 1998. 